Bundesgesundheitsbl $2011 \cdot 54: 244-244$ DOI 10.1007/s00103-010-1212-y

Online publiziert: 29. Januar 2011

(c) Springer-Verlag 2011

\title{
5. Mitteilung zur Änderung der Leitlinie zur hygienischen Bewertung von Schmierstoffen im Kontakt mit Trinkwasser (Sanitärschmierstoffe) [Schmierstoffleitlinie] ${ }^{1}$
}

Der Schmierstoff „Castrol Optisil Leb 2 “ der Firma „Deutsche BP Aktiengesellschaft" wird für die Temperaturbereiche Kalt- und Warmwasser mit einer Gültigkeit bis 01.02.2012 aufgenommen.

Der Schmierstoff „Chemplex 746 (\#169) weiß“ der Firma „Fuchs Lubri-

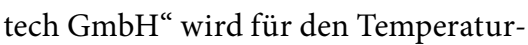
bereich Kaltwasser mit einer Gültigkeit bis 14.12.2012 aufgenommen.

Der Schmierstoff „Chemplex SI 410 (medium)“ der Firma „Fuchs Lubritech $\mathrm{GmbH}$ “ wird für den Temperaturbereich Kaltwasser mit einer Gültigkeit bis 10.07.2013 aufgenommen.

Der Schmierstoff „Geralyn 2 “ der Firma „Fuchs Lubritech GmbH“ wird für den Temperaturbereich Kaltwasser mit einer Gültigkeit bis 13.02.2012 aufgenommen.

Der Schmierstoff „Gleitmo 815“ der Firma „Fuchs Lubritech GmbH“ wird für den Temperaturbereich Kaltwasser mit einer Gültigkeit bis 22.10.2012 aufgenommen.
Folgende Schmierstoffe werden gestrichen:

Armaturenfett „diamant Type 4" der Firma „Artur Glöckler GmbH“, „Molyduval Silikonfett GO“ der Firma „Molyduval von Laar GmbH“ und „Winix 2300“ der Firma „Winix GmbH“.

1 zuletzt geändert: Bundesgesundheitsbl Gesundheitsforsch Gesundheitsschutz 2009 52:965 